\title{
MATRIX REPRESENTATIONS OF $d$-SIMPLE SEMIGROUPS
}

\author{
BY \\ R. J. WARNE
}

By a 'representation' we shall mean throughout a representation by $n \times n$ matrices with entries from an arbitrary (commutative) field.

Clifford has constructed all representations of completely simple semigroups $[1 ; 4]$. Munn has determined the representations of finite semigroups for which the corresponding semigroup algebra is semi-simple [6]. It is noted by Clifford and Preston [4] that if $S$ is a semigroup satisfying the descending chain condition for principal ideals and such that every 0 -simple principal factor is completely 0 -simple then all the irreducible representations of $S$ can be expressed in terms of those of subgroups of $S$. This statement is a consequence of work of Clifford [1] and Munn [4]. Munn has determined the irreducible representations of intraregular inverse semigroups [7].

In $\S 1$, we prove the following result:

Let $S$ be a semigroup having a maximal group homomorphic image $G$. Then there is a one-to-one correspondence between the representation of $G$ and the nonsingular representations of $S$ which preserves equivalence, reduction and decomposition (Theorem 1.2). An application of this result to inverse semigroups is given. Stoll [11] gives some examples of semigroups with maximal group homomorphic images.

In $\$ 2$, we determine the representations of an important class of $d$-simple semigroups by utilizing Theorem 1.2.

Let $S$ be a semigroup satisfying the following conditions:

(A1) $S$ is $d$-simple.

(A2) $S$ has an identity element.

(A3) Any two idempotents of $S$ commute.

It is shown by Clifford [2] that the structure of $S$ is determined by that of its right unit semigroup $P$ and that $P$ has the following properties:

(B1) The right cancellation law holds in $P$.

(B2) $P$ has an identity element, 1.

(B3) The intersection of two principal left ideals of $P$ is a principal left ideal. Condition (B3) implies that for any $a, b$ in $P$ there exists $x$ and $y$ in $P$ such that

Presented to the Society, November 16, 1962, November 24, 1962, under the title Matrix representations of d-simple semi-groups. I, II; received by the editors March 4, 1962. 
$x a=y b$, i.e. any two elements of $P$ have a common left multiple. We call this property (B3)'.

We define a congruence relation $\eta$ on $P$ such that $\bar{P}=S / \eta$ satisfies (B1), (B2), (B3)' and the left cancellation law. Thus, as shown by Ore [8; 4, p. 34], $\bar{P}$ may be embedded in a group $G$ of "fractions." We show that there exists a one-to-one correspondence between the representations of $S$ and the representations of $G$ which preserves equivalence, reduction, and decomposition. We also give an explicit formula for constructing the representations of $S$ in terms of those of $G$. In $\S 3$, we apply this formula to determine the representations of the bicyclic semigroup and those of a more complicated semigroup.

We wish to express our gratitude to Professor A. H. Clifford for providing an alternative treatment leading to our main result (Theorem 2.2).

This treatment, which we give here, considerably reduces the length of the original paper. We also wish to thank Professor Clifford for making available to us, before publication, Chapter 5 of his book [4].

1. Nonsingular representations. A semigroup is a set which is closed under an associative binary operation. This, as usual, will be denoted by juxtaposition.

A nonvoid subset $A$ of a semigroup $S$ is an ideal of $S$ if and only if $S A \subseteq A$ and $A S \subseteq A$.

A semigroup $S$ is simple if and only if $S$ is its only ideal and it is not the null semigroup of order one.

To each element $a$ of a semigroup $S$ let there correspond a uniquely determined matrix $T(a)$ with $n$ rows and columns with elements in a (commutative) field $\Omega$. If for all $a, b$ in $S$

$$
T(a b)=T(a) T(b)
$$

then the correspondence $T: a \rightarrow T(a)$ is called a (matrix) representation of $S$ in $\Omega$ of degree $n$. A representation $T$ of a semigroup $S$ will be called nonsingular if and only if $T(a)$ is a nonsingular matrix for all $a$ in $S$.

The notions of equivalence, reduction, and decomposition are defined exactly as in the theory of representations of groups or algebras.

A representation $T$ of a semigroup without zero is proper if and only if $T$ is not decomposed into two representations one of which is null and $T$ is not the null representation of degree one. There is no essential loss of generality in confining our attention to proper representations.

THEOREM 1.1. Let $S$ be a simple semigroup with identity element 1. Then every proper representation of $S$ is nonsingular.

Proof. If $T$ is a proper representation of $S$ of degree $n$, then $T(1)=I n$. If $a$ is in $S$, then there exists $x, y$ in $S$ such that $x a y=1[4 ; 9]$, since $S$ is simple, and from $T(x) T(a) T(y)=I n$, it follows that $T(a)$ is nonsingular. 
A semigroup $S$ will be said to have a maximal group homomorphic image $G$ if there exists a homomorphism $\phi$ of $S$ onto $G$ such that if $\tau$ is any homomorphism of $S$ into a group $H$, then there is a homomorphism $\tau^{*}$ of $G$ into $H$ such that $\tau^{*} \phi=\tau$ (first $\phi$ then $\tau^{*}$ ).

Since $\phi$ is onto, the correspondence $\tau \leftrightarrow \tau^{*}$ is one-to-one, each uniquely determining the other.

THEOREM 1.2. Let $S$ be a semigroup having a maximal group homomorphic image G. Then there is a one-to-one correspondence between the (proper) representations of $G$ and the nonsingular representations of $S$ which preserves equivalence, reduction, and decomposition.

Proof. Taking $H$ to be the group of all nonsingular $n \times n$ matrices over a field $\Omega$, it follows that there is a one-to-one correspondence $T \leftrightarrow T^{*}$ between the nonsingular representations $T$ of $S$ and the (proper) representations $T^{*}$ of $G$, whereby, for each $a$ in $S$,

$$
T(a)=T^{*}(\phi(a)) .
$$

It is easily seen that this preserves equivalence. To show it preserves reduction, let $T \leftrightarrow T^{*}$ be corresponding representations of degree $n$, and consider $n \times n$ matrices as linear transformations of an $n$-dimensional vector space $V$. If $W$ is a subspace of $V$ invariant under $T^{*}$, then

$$
T(a) w=T^{*}(\phi(a)) w \text { in } W \text { (for all } w \text { in } W \text { and } a \text { in } S \text { ), }
$$

so $W$ is also invariant under $T$. The converse is true since $\phi$ is onto. If $T_{1}$ and $T_{1}^{*}$ are the representations of $S$ and $G$ respectively induced by $T$ and $T^{*}$ in $W$ (namely just their restrictions to $W$ ) then trivially

whence

$$
T_{1}(a) w=T_{1}^{*}(\phi(a)) w \quad(\text { for all } w \text { in } W \text { and } a \text { in } S)
$$

$$
T_{1}(a)=T_{1}^{*}(\phi(a)) \quad \text { for all } a \text { in } S \text {, i.e. } T_{1} \leftrightarrow T_{1}^{*} .
$$

Let $T_{2}$ and $T_{2}^{*}$ be the representations of $S$ and $G$ respectively induced by $T$ and $T^{*}$ in the factor space $V-W$; that is

and

$$
T_{2}(a)(v+W)=T(a) v+W \quad(\text { for all } v \text { in } V \text { and } a \text { in } S)
$$

We then have

$$
T_{2}^{*}(\alpha)(v+W)=T^{*}(\alpha) v+W \quad(\text { for all } v \text { in } V \text { and } \alpha \text { in } G) .
$$

$$
\begin{aligned}
T_{2}(a)(v+W) & =T(a) v+W=T^{*}(\phi(a)) v+W \\
& =T_{2}^{*}(\phi(a))(v+W) \quad(\text { for all } v \text { in } V \text { and } a \text { in } S) .
\end{aligned}
$$

Thus,

whence $T_{2} \leftrightarrow T_{2}^{*}$.

$$
T_{2}(a)=T_{2}^{*}(\phi(a)) \quad(\text { for all } a \text { in } S)
$$


This shows that reduction is preserved and decomposition is similar (if not actually a consequence); for if $V=W_{1} \oplus W_{2}$ with $W_{1}$ and $W_{2}$ invariant under $T$, then they are invariant under $T^{*}$ (and vice-versa) and the representations $T_{i}$ and $T_{i}^{*}$ induced in $W_{i}(i,=1,2)$ correspond as above. q.e.d.

A semigroup $S$ is an inverse semigroup if and only if $a$ in $a S a$ for all $a$ in $S$ and the idempotents of $S$ commute [5]. Let $S$ be an inverse semigroup and let a relation $\sigma$ be defined on $S$ by the rule $x \sigma y$, if and only if there exists an idempotent $e$ in $S$ such that $e x=e y$. It follows easily from a theorem of Munn [7, p. 42] that $G=S / \sigma$ is a maximal group homomorphic image of $S$. Thus, by Theorem 1.2 there exists a one-to-one correspondence $T^{*} \leftrightarrow T$ between the representations of $G$ and the nonsingular representations of $S$ which preserves equivalence, reduction and decomposition, and $T(a)=T^{*}(\bar{a})$ where $a \rightarrow \bar{a}$ is the natural homomorphism of $S$ onto $G$. We note that not all proper (or even all irreducible) representations of $S$ are nonsingular. For let $S$ be the semigroup which consists of the real interval $[0,1]$ with multiplication defined by

Then,

$$
x y=\max \{x, y\} .
$$

$$
T a(x)=\left\{\begin{array}{l}
1 \text { if } x \leqq a \\
0 \text { otherwise }
\end{array} \quad(0<a<1)\right.
$$

is an irreducible representation of $S$.

2. Matrix representations of $d$-simple semigroups. We first summarize the construction of Clifford referred to in the introduction.

Let $S$ be any semigroup with identity element. We say that two elements are right associate if they generate the same principal right ideal: $a S=b S$. Left associate elements are defined analogously. Two elements $a$ and $b$ are called $d$-equivalent if there exists an element of $S$ which is left associate to $a$ and right associate to $b$. (This implies the existence of an element of $S$ which is right associate to $a$ and left associate to $b$.) We shall say that $S$ is $d$-simple if it consists of a single class of $d$-equivalent elements.

Now let $P$ be any semigroup satisfying (B1), (B2), and (B3). From each class of left associate elements of $P$ let us pick a fixed representative. (B3) states that if $a$ and $b$ are elements of $P$, there exists $c$ in $P$ such that $P a \cap P b=P c$. $c$ is determined by $a$ and $b$ only to within left dssociates. We define $a \vee b$ to be the representative of the class to which $c$ belongs. We observe also that

$$
a \vee b=b \vee a \text {. }
$$

We define a binary operation $x$ by

$$
(a \times b) b=a \vee b
$$

for each pair of elements $a, b$ of $P$. 
Now let $P^{-1} \circ P$ denote the set of ordered pairs $(a, b)$ of elements of $P$ with equality defined by

$$
(a, b)=\left(a^{\prime}, b^{\prime}\right) \text { if } a^{\prime}=u a \text { and } b^{\prime}=u b
$$

where $u$ is a unit in $P$ ( $u$ has a two sided inverse with respect to 1 the identity of $P$ ).

We define product in $P^{-1} \circ P$ by

$$
(a, b)(c, d)=((c \times b) a,(b \times c) d) .
$$

Clifford's main theorem states: Starting with a semigroup $P$ satisfying (B1), (B2), (B3) equations (2.2), (2.3), and (2.4) define a semigroup $P^{-1} \circ P$ satisfying (A1), (A2), (A3). $P$ is isomorphic with the right unit subsemigroup of $P^{-1} \circ P$ (the right unit subsemigroup of $P^{-1} \circ P$ is the set of elements of $P^{-1} \circ P$ having a right inverse with respect to 1 . This set is shown early to be a semigroup). Conversely, if $S$ is a semigroup satisfying (A1), (A2), (A3), its right unit subsemigroup $P$ satisfies (B1), (B2), (B3) and $S$ is isomorphic with $P^{-1} \circ P$. The following results are also obtained:

The elements $(1, a)$ of $P^{-1} \circ P$ constitute a subsemigroup thereof isomorphic to $P$. We have

$$
(1, a)(1, b)=(1, a b) \text { for } a, b \text { in } P .
$$

The ordered pair $(1,1)$ is the identity of $P^{-1} \circ P$, i.e.

$$
(a, b)(1,1)=(1,1)(a, b)=(a, b) \text { for } a, b \text { in } P .
$$

The right inverse of $(1, a)$ is $(a, 1)$, i.e.

$$
(1, a)(a, 1)=(1,1) \text { for } a \text { in } P \text {. }
$$

Finally

$$
(a, c)=(a, 1)(1, c) \text { for all } a \text { and } c \text { in } P \text {. }
$$

For the remainder of this section, we identify $S$ with $P^{-1} \circ P$ and $P$ with $\{(1, a): a$ in $P\}$.

Now, as noted in the introduction, $P$ satisfies (B1), (B2), and (B3)' ${ }^{\prime}$. We now introduce the following relation on $P$ :

$$
a \eta b \text { if and only if there exists } h \text { in } P \text { such that } h a=h b \text {. }
$$

It is easily seen that $\eta$ is an equivalence relation. We next show that $a \eta b$ implies $c a \eta c b$ for all $c$ in $P$. Since $a \eta b$, there exists $h$ in $P$ such that $h a=h b$. By (B3)', there exists $x$ and $y$ in $P$ such that $x c=y h=k$. Hence, $k a=k b$ and $x c a=x c b$. Clearly, $a c \eta b c$ for all $c$ in $P$. Thus, $\eta$ is a congruence relation. Let $a \rightarrow \bar{a}$ be the natural homomorphism of $P$ onto $\bar{P}$, the semigroup of congruence classes. Clearly $\bar{P}$ satisfies (B1), (B2), (B3)' and the left cancellation law. We may rewrite (2.9) as

$$
\bar{a}=\bar{b} \text { if and only if there exists } h \text { in } P \text { such that } h a=h b \text {. }
$$


(The right-left dual of the above is given by Skolem [10].)

As shown by Ore $[8 ; 4$, p. 34$], \bar{P}$ may be embedded in a group $G$. We will briefly review this construction.

Let $\bar{a}, \bar{b}$ be in $\bar{P}$. We consider the set $G$ of all pairs of elements of $\bar{P}$ writing them as fractions $\bar{b} / \bar{a}$. The relation $=$ between these fractions shall be defined thus:

$$
\bar{b} / \bar{a}=d / \bar{c}
$$

shall mean that elements $\bar{x}$ and $\bar{y}$ exist in $\bar{P}$ such that

$$
\bar{x} \bar{a}=\bar{y} \bar{c} \text { and } \bar{x} \bar{b}=\bar{y} d .
$$

The definition of the product is

where $h \bar{b}=k \bar{c}$.

$$
\bar{b} / \bar{a} d / \bar{c}=\bar{k} d / h \bar{a}
$$

The multiplication is associative, $\overline{1} / \overline{1}(\overline{1}$ is the identity of $\bar{P})$ is the identity, $\bar{b} / \bar{a}$ is the inverse of $\bar{a} / \bar{b}$, i.e. $G$ is a group. The isomorphism of $\bar{P}$ into $G$ is given by $\bar{a} \rightarrow \bar{a} / \overline{1}$.

Since we have succeeded in embedding $\bar{P}$ in the group $G$, it is no longer necessary to retain the quotient notation. The final result is that $\bar{P}$ is a subsemigroup of $G$ and every element of $G$ is expressible in the form $\bar{a}^{-1} \bar{b}$ with $\bar{a}$ and $\bar{b}$ in $\bar{P}$. For future reference, we rewrite (2.11) as

$$
\bar{a}^{-1} \bar{b}=\bar{c}^{-1} d
$$

if and only if there exists $\bar{x}$ and $\bar{y}$ in $\bar{P}$ such that

$$
\bar{x} \bar{a}=\bar{y} \bar{c} \text { and } \bar{x} \bar{b}=\bar{y} d \text {. }
$$

THEOREM 2.1. With $S, P, \bar{P}$, and $G$ as above, the mapping $\phi:(a, b) \rightarrow \bar{a}^{-1} \bar{b}$ is $a$ homomorphism of $S$ onto $G$, and $G$ is thereby a maximal homomorphic image of $S$.

Proof. To show that $\phi$ is single valued, let $(a, b)=\left(a^{\prime}, b^{\prime}\right)$. Then, $a^{\prime}=u a$ and $b^{\prime}=u b$ for some unit $u$ in $P$ by (2.3). This implies $\bar{a}^{\prime}=\bar{u} \bar{a}, b^{\prime}=\bar{u} \bar{b}$, and hence

$$
\bar{a}^{-1} \bar{b}^{\prime}=\bar{a}^{-1} \bar{u}^{-1} \bar{u} \bar{b}=\bar{a}^{-1} \bar{b} \text {. }
$$

To show that $\phi$ is a homomorphism, we first note that from (2.2) and (2.1) we have

Hence,

$$
(b \times c) c=b \vee c=c \vee b=(c \times b) b .
$$

and so

$$
\overline{b \times c} \cdot \bar{c}=\overline{c \times b} \cdot \bar{b}
$$

for any $b, c$ in $P$. 
Hence, using (2.4) and (2.13) we obtain

$$
\begin{aligned}
\phi[(a, b)(c, d)] & =\phi((c \times b) a,(b \times c) d) \\
& =\overline{(c \times b) a}-1 \overline{(b \times c) d}=\bar{a}^{-1} \overline{c \times b}-1 \overline{b \times c} d \\
& =\bar{a}^{-1} \bar{b} \bar{c}^{-1} d=\phi(a, b) \cdot \phi(c, d) .
\end{aligned}
$$

We next show that if $\tau$ is any homomorphism of $S$ into a group $H$,

$$
\phi(a, b)=\phi(c, d) \text { implies } \tau(a, b)=\tau(c, d) .
$$

From, $\phi(a, b)=\phi(c, d)$, we have

$$
\bar{a}^{-1} b=\bar{c}^{-1} d .
$$

Hence, by (2.12), $\bar{x} \bar{a}=\bar{y} \bar{c}$ and $\bar{x} \bar{b}=\bar{y} d$ for some $\bar{x}, \bar{y}$ in $\bar{P}$. Therefore, $\overline{x a}=\overline{y c}$ and $\overline{x b}=\overline{y d}$, so $f x a=f y c$ and $g x b=g y d$ for some $f, g$ in $P$ by (2.10). Hence

$$
\begin{aligned}
& (1, f)(1, x)(1, a)=(1, f)(1, y)(1, c), \\
& (1, g)(1, x)(1, b)=(1, g)(1, y)(1, d),
\end{aligned}
$$

and, applying $\tau$ and cancelling $\tau(1, f)$ and $\tau(1, g)$,

$$
\begin{aligned}
\tau(1, x) \tau(1, a) & =\tau(1, y) \tau(1, c), \\
\tau(1, x) \tau(1, b) & =\tau(1, y) \tau(1, d) .
\end{aligned}
$$

The foregoing are equations in the group $H$, and so

$$
\tau(1, a)^{-1} \tau(1, b)=\tau(1, c)^{-1} \tau(1, d) .
$$

From equation $(2.7),(1, a)(a, 1)=(1,1)$, we have

$$
\tau(1, a)^{-1}=\tau(a, 1)
$$

and so the above becomes

$$
\tau(a, 1) \tau(1, b)=\tau(c, 1) \tau(1, d)
$$

or using (2.8),

$$
\begin{aligned}
\tau(a, b) & =\tau[(a, 1)(1, b)]=\tau(a, 1) \tau(1, b) \\
& =\tau(c, 1) \tau(1, d)=\tau[(c, 1)(1, d)]=\tau(c, d) .
\end{aligned}
$$

This concludes the proof of (2.14).

If $\alpha$ is in $G$, we define $\tau^{*}$ by

$$
\tau^{*}(\alpha)=\tau(a, b) \text { where } \phi(a, b)=\alpha
$$

in order to make $\tau^{*} \phi=\tau$. This definition of $\tau^{*}$ is single valued, for if $\alpha=\phi(c, d)$ then $\tau(a, b)=\tau(c, d)$ by (2.14). Thus, $\tau^{*}$ is (uniquely) defined by

$$
\tau^{*}[\phi(a, b)]=\tau(a, b) .
$$


This automatically makes $\tau^{*}$ a homomorphism of $G$ into $H$ :

$$
\begin{aligned}
\tau^{*}[\phi(a, b) \phi(c, d)] & =\tau^{*}[\phi\{(a, b)(c, d)\}] \\
& =\tau\{(a, b)(c, d)\}=\tau(a, b) \tau(c, d) \\
& =\tau^{*}[\phi(a, b)] \tau^{*}[\phi(c, d)] .
\end{aligned}
$$

(Recall that $\phi$ is onto.) q.e.d.

Since a $d$-simple semigroup with zero consists of zero alone we may eliminate these semigroups from our discussion.

THEOREM 2.2. Let $S, P, \bar{P}$, and $G$ be defined as above. Then there is a correspondence $T \leftrightarrow T^{*}$ between the proper representations of degree $n$ of $S$ and the (proper) representations of degree $n$ of $G$ given by

$$
T(a, b)=T^{*}(\bar{a})^{-1} T^{*}(\bar{b})
$$

where $a \rightarrow \bar{a}$ is the natural homomorphism of $P$ onto $\bar{P}$ given on $p .431$. This correspondence is one-to-one and preserves equivalence, reduction, and decomposition.

Proof. A representation of $S$ is proper if and only if it is nonsingular by Theorem 1.1. Hence, by virtue of Theorem 2.1 the construction presented in the proof of Theorem 1.2 is applicable. Thus, taking $\phi(a b)=\bar{a}^{-1} \bar{b}$,

$$
T[(a, b)]=T^{*}[\phi(a, b)]=T^{*}\left[\bar{a}^{-1} \bar{b}\right]=T^{*}(\bar{a})^{-1} T^{*}(\bar{b})
$$

where $T, T^{*}$ are the corresponding representations of $S$ and $G$ respectively.

REMARK 1. A semigroup $S$ is intraregular if and only if $a$ is in $S a^{2} S$ for all $a$ in $S$. The class of $d$-simple semigroups we consider are intraregular [3, p. 502]. It follows from (2.8), (2.7), and (A3) that they are inverse semigroups. Hence their irreducible representations may be determined from the work of Munn [7].

REMARK 2. We note that our paper is the first paper in matrix representations of semigroups that makes use of the technique of embedding a semigroup in a more special semigroup. We feel this technique may have further applications in the representation theory.

3. Examples. We give two examples illustrating the application of (2.15).

EXAMPLE 1. The bicyclic semigroup " $C$ " $[4$, p. 43] consists of all pairs of non-negative integers with multiplication given by:

$$
(i, j)(k, s)=(i+k-\min (j, k), j+s-\min (j, k)) .
$$

A complete set of proper representations of " $C$ " of degree $n$ is given by

$$
T_{A}(i, j)=A^{-i+j} \quad(i, j \text { are non-negative integers })
$$

where $A$ runs through all nonsingular matrices of degree $n$. 
EXAMPLE 2. Let $G$ be any group of order greater than or equal to two with identity $e$. Let $I_{0}$ be the non-negative integers under the usual addition.

Consider $P=G \times I_{0}$ under the following multiplication.

$$
(u, s)(v, k)= \begin{cases}(u v, k) & \text { if } s=0 \\ (u, s+k) & \text { if } s \neq 0\end{cases}
$$

$P$ is a semigroup satisfying (B1), (B2), and (B3) which is not left cancellative. Let $S$ be the semigroup corresponding to $P$ in Clifford's main theorem (see introduction).

A complete set of proper representations of $S$ of degree $n$ is given by

$$
T_{A}[(i, j),(k, s)]=A^{-j+s}((i, j) \text { and }(k, s) \text { are in } P)
$$

where $A$ runs through all nonsingular matrices of degree $n$.

\section{REFERENCES}

1. A. H. Clifford, Matrix representations of completely simple semigroups, Amer. J. Math. 64 (1942), 327-342.

2. — $-A$ class of $d$-simple semigroups, Amer. J. Math. 75 (1953), 547-556.

3. - Bands of semigroups, Proc. Amer. Math. Soc. 5 (1954), 499-504.

4. A. H. Clifford and G. B. Preston, The algebraic theory of semigroups, Math. Surveys No. 7, Amer. Math. Soc., Providence, R. I., 1961.

5. W. D. Munn and R. Penrose, $A$ note on inverse semigroups, Proc. Cambridge Philos. Soc. 51 (1955), 396-399.

6. W. D. Munn, Matrix representations of semigroups, Proc. Cambridge Philos. Soc. 53 (1957), 5-12.

7. - A class of irreducible matrix representations of an arbitrary inverse semigroup, Proc. Glasgow Math. Assoc. 5 (1961), 41-48.

8. O. Ore, Linear equations in non-commutative fields, Ann. of Math. 32 (1931), 463-477.

9. D. Rees, On semigroups, Proc. Cambridge Philos. Soc. 36 (1940), 387-400.

10. Th. Skolem, Some remarks on semigroups, Norske Vid. Selsk. Forh. Trondheim 24 (1951), 42-47.

11. R. R. Stoll, Homomorphisms of a semigroup onto a group, Amer. J. Math. 73 (1951), $475-481$.

Louisiana State University in New Orleans, New OrLeans, Louisiana 